\title{
A Knowledge-intensive Serious Game for Education of Traditional Chinese Medicine: A Pilot Study
}

\author{
Youliang Huang ${ }^{1,2}$, Renquan Liu ${ }^{1, *}$, Shuangqing Zhai ${ }^{1}$, Xingguang $\mathrm{Ma}^{1}$, Mingquan \\ Zhou $^{2}$ and Qingqiong Deng ${ }^{2}$ \\ ${ }^{1}$ Information Center, Beijing University of Chinese Medicine, China \\ ${ }^{2}$ College of Information Science \& Technology, Beijing Normal University, China \\ Correspondence author: liurq@bucm.edu.cn
}

\begin{abstract}
Traditional Chinese Medicine (TCM) plays an important role in South-East Asia, and increasingly in the West. However, learning and mastering TCM knowledge are still an obscure problem for researchers all over the world, especially for beginners. With the development of computer science, serious game rising in a wide range of fields like education, training and simulation, etc. In this paper, we design and implementation a knowledge-intensive serious game for education of TCM. When the users play this game, they will finding learning is an interesting thing. We chose the Unity $3 D$ as our game engine, $3 d s$ Max 2013 as model design tools. When the users log in the game, it will presents a series of scenes include some tasks and questions about the TCM knowledge. After a series tasks and test, the reslut shows that serious game for TCM education proved effective to help the user remember and comprehend the concept of TCM.
\end{abstract}

Keywords: Serious game, Traditional Chinese Medicine education, Computer-aid education

\section{Introduction}

Traditional Chinese Medicine (TCM) has been used in China as the predominant medicine and plays an important role in South-East Asia, and increasingly in the West [1]. Because of the complexity and vastness of TCM, it is still an obscure problem for researchers all over the world, especially for beginners. It is the most important issue in TCM education that the fragments of literature work into a unified reusable and analyzable knowledge system.

Serious (computer-based) gaming is a novel technique in many fields, which has the potential to become an important learning tool for education for learning a range of knowledge. The term serious game [2] refers to games designed to do more than just entertain. Rather, serious games, while having many features in common with more traditional games, have ulterior motives such as teaching, training, and marketing. The previous studies have demonstrated that serious gaming is a stimulating learning method and that students are enthusiastic about its use.

In this paper, we describe a knowledge-intensive game for TCM education. The aim of our study was to design and development a serious game as a novel tool for TCM education. Our game integrates the TCM knowledge according to the story-line of the game.

The remaining of the paper is organized as follows. We first review applications of serious games in various disciplines in Section II. Then, we present our game design specifications in Section III. Experiments and results are reported in Section IV, followed by discussion and conclusions in Section V and Section VI, respectively..

\section{Literature Review}

In this section, we performed a systematic literature review. With the development of game industry and the popularity of computer-aided instruction, it is currently possible 
to find a large number of research aimed at applying virtual reality [3] and immersive virtual reality $[4,5]$ to the education of students with special educational needs. As the product of education and games, serious game will improve the traditional teaching mode [6-8], and integrate more vivid teaching form and richer teaching contents into it, to realize the educational thoughts of "learning with entertainment". As M. Prensky recounts in his book [2], "The true 21 st century learning revolution is that, learning through playing and playing to learn."

The serious game for education has an early start. In 1970, Clark Abt discussed the term in his book "Serious Games" [9], which was primarily dedicated to the use of board and card games. His general definition which is still considered applicable in the computer age which is: "Reduced to its formal essence, a game is an activity among two or more independent decision-makers seeking to achieve their objectives in some limiting context". In the $1980 \mathrm{~s}$, some researchers proposed that the concept of "educational game", that is serious game for education, in the course of researching the video game in America [10-12]. In the development theory of the serious game, west researchers have done a lot of work, and proposed some development models and methods. A more conventional definition was introduced by Mike Zyda in 2005 [13] to provide an updating and more complete definition to the term as: "A mental contest, played with a computer in accordance with specific rules that uses entertainment to fulfill government or corporate training, education, health, public policy, and strategic communication objectives." At the same time, Stoke postulated a new definition for serious gaming: 'games that are designed to entertain players as they educate, train, or change behavior' $[14,15]$, in 2005 . Nowadays, the term is used to cover a spectrum of computer-based simulations for training or education in a single or multi-user environment.

In education theory, Knowles described four elements important to adult learning: 1) Adults are autonomic and want independence in their learning. Gaming promotes an active form of learning and allows independence [16]. 2) Adults use their past experience. Gaming facilitates this by offering different scenarios according to experience [17, 18]. 3) Adults are goal-orientated. Gaming is designed around completion of a level or task. 4) Adults tend to be problem-based learners and not content-orientated learners [19]. Gaming provides learning experiences that players can relate to realistic clinical problems. Other theories also support serious gaming as a method for the education and training. Serious games have been used in particular education for a long time.

However, there are several criticisms made of serious gaming as a method for learning and training. One common criticism is that the dynamic colorful world of a computer game will distract the student attention from the learning process. Prensky and other proponents of serious gaming believe that the present generation contains a different type of learner, and probably it was ever thus [20-22]. Current and future professionals grew up with digital media and have developed a much better aptitude to relate a virtual world to reality $[23,24]$. Nevertheless, for serious gaming to become accepted, these criticisms must be addressed [25], as much as the need to demonstrate the clinical effectiveness of serious gaming [26, 27]. There is some evidence that the serious game for education is a suitable and beneficial approach to foster learning motivation [28], engagement with a subject, and to improve the attitude towards a subject $[29,30]$. 


\section{Methods}

\subsection{Aims of the Study}

The overall objective of this study is to create a knowledge-intensive game for TCM education, using computer-aid technology. This general approach can be broken down into several specific objectives, as described below:

1) Design a game aimed at learn TCM knowledge in a fun environment;

2) Develop an interface that suits the needs of TCM education and makes it easier to use;

3) Stimulate interest in learning and positive thinking.

\subsection{System Architecture}

The purpose of all the study is to generate a TCM education game. The system architecture includes three layers: presentation layer, logic layer and data layer.

1) Presentation layer is mainly used for interface display and to achieve the user's query.

2) Logic layer control the game workflow.

3) Data layer is mainly store the user data, game data and various TCM acknowledgements. The architecture of the game is show in Figure 1.

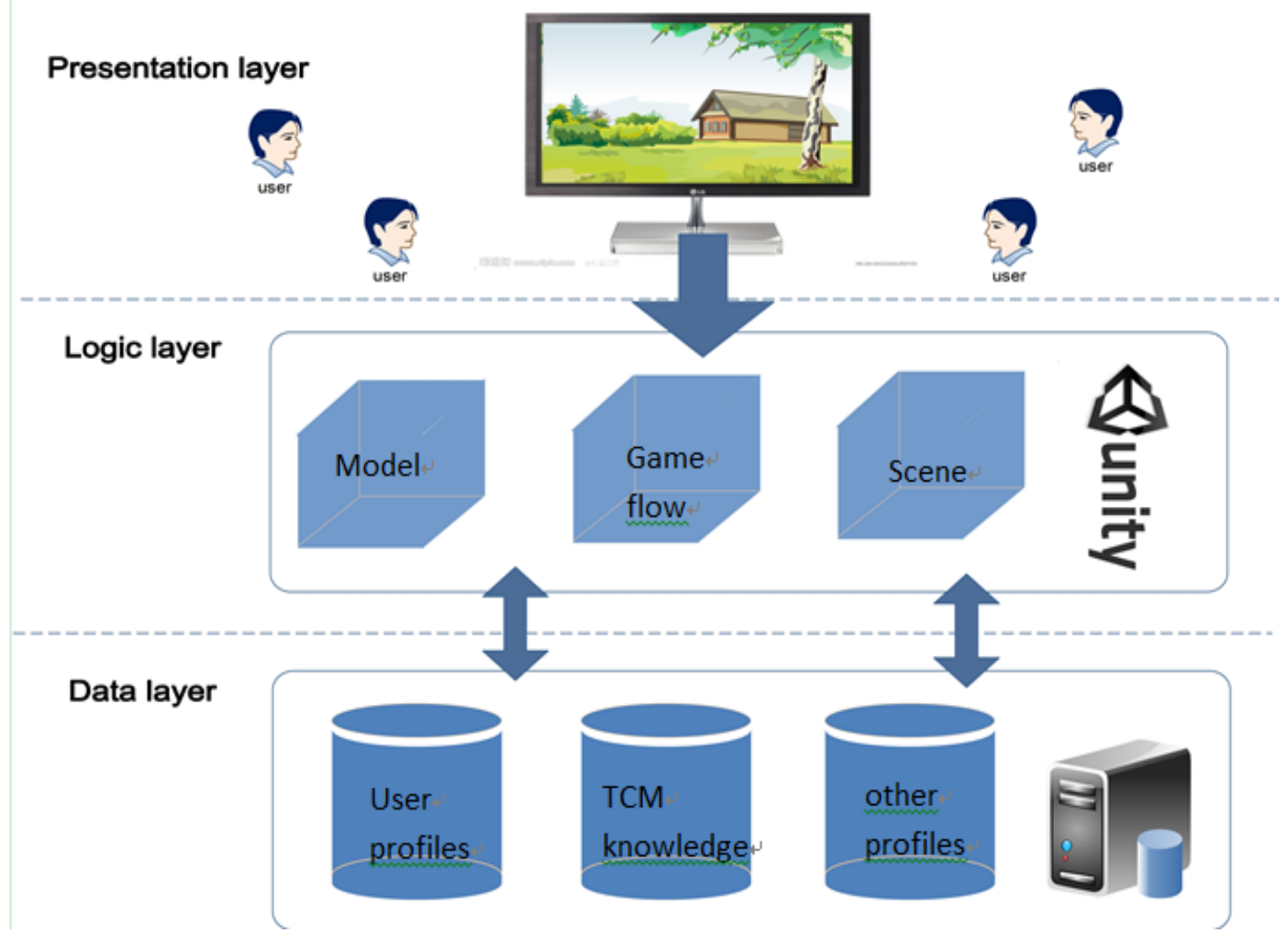

Figure 1. The Architecture of the Game for TCM Education

\subsection{Implementation}

3.3.1. Game Engine: Game engine is a game development platform. It is a set of tools that provide necessary features to the development of serious games. After comparing various game engines, Unity3D [31, 32] was selected. This study is implemented based on Unity3D game engine. Unity3D is a popular one, which also supports the development of plug-in. The specific features of Unity3D include an integrated editor, 
cross-platform release, terrain editing, shading, scripts, networking, physics, version control and other features. It supports three scripting languages: JavaScript, C\# and a dialect of Python called Boo. These three languages in game development projects can also be mixed-use. It also supports the Windows platform, C\#, VB.net, VB6, Delphi and other programming languages [6].

3.3.2. 3D Model: 3D modeling is an important piece of the TCM game. There are different tools to generated 3D models and virtual scenes, such as 3ds Max [33], Maya [34] and Blender [35].The choice of modeling system dictates what type of geometry will appear in the game. In this paper, the main tools for modeling are $3 \mathrm{ds}$ Max 2013. The game models and scenes consist of avatar, drugstore, study, buildings and so on. The screenshot of the model in the game is shown in Figure 2.

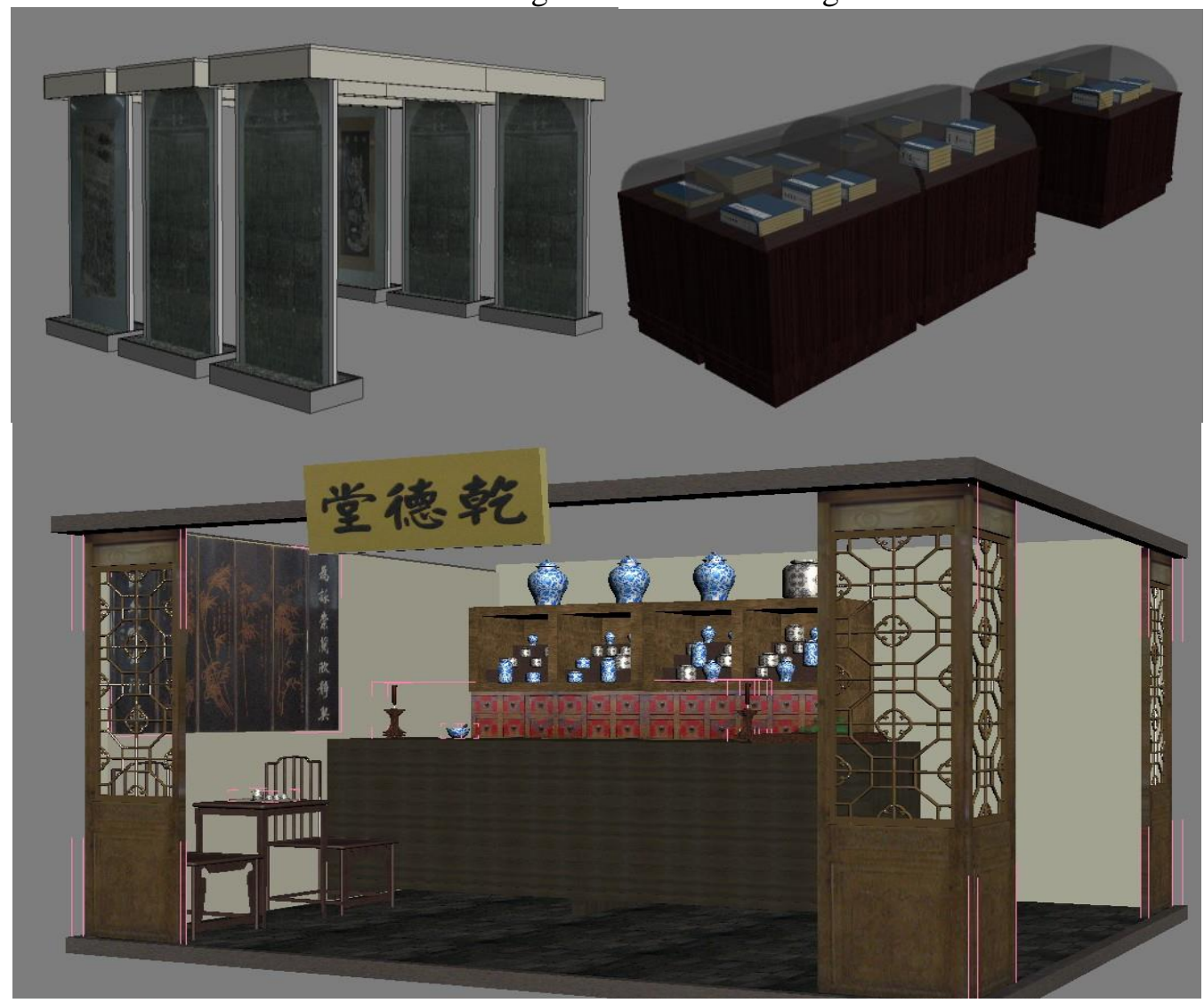

Figure 2. The Screenshot of the Scene

3.3.3. Game Design: In order to adapt the different TCM knowledge, we had to simplify the game level. According to the story-line, we design ten levels. Every level has fixed learning content and aim. Table 1 shows the detail.

Table 1. The Levels of the Game

\begin{tabular}{lll}
\hline Number & Content & Aim \\
\hline The first level & The origin of TCM & Understand the origin of TCM \\
The second level & TCM culture & Learn and master some important TCM culture \\
The third level & TCM herb culture & Learn more about the common TCM herd
\end{tabular}




\begin{tabular}{|c|c|c|}
\hline The fourth level & TCM school & Remember TCM school and their years \\
\hline The fifth level & TCM doctors in the history & Learn and remember the famous TCM doctors \\
\hline The sixth level & TCM classics & Collect ancient TCM literatures and copy them \\
\hline The seventh level & TCM and modern medicine & Differentiate TCM and modern medicine \\
\hline The eighth level & TCM exercise prescription & Study and remember TCM prescription \\
\hline The ninth level & TCM clinic & Learn and study TCM clinic and try to apply \\
\hline The tenth level & Comprehensive application & The phase review some key content \\
\hline
\end{tabular}

In the first and second level, we select traditional Chinese culture as the background. The task is to understand and learning culture of the traditional Chinese medicine. In the third and fourth level, the task is to learn the growth story of the famous TCM doctor in the study scene. In the fifth and sixth level, we select study as the main scene, the task is the user must read and remember the explanations of ancient TCM classics. In the nine and ten level, the user finishes his intermediate stage of the study; he can diagnose the simple illness. The game allowed the user interaction (via dialog, task, audio, exam, etc.,) and can reach a higher level after they complete a task in the game. Figure 3 shows the flow of the game.

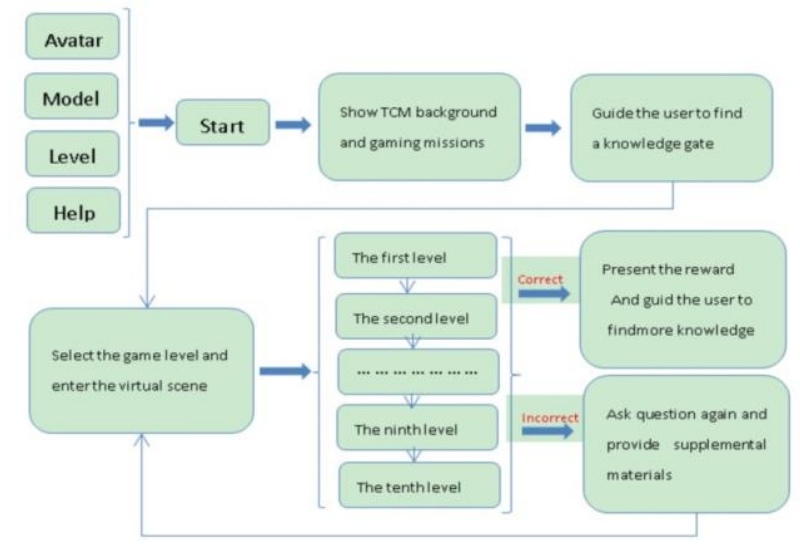

Figure 3. The Flow of the Game

\section{Main Title Experiment and Results}

According to the above method, we design and development the game for TCM education. In order to test our approach and evaluate its potentials, a simple experiment was executed and some results analyzed. The hardware consisted of a computer with an Intel Core Quad CPU Q6700, 4GB of RAM, and a GeForce 8800GT and the operate system is Windows 7 SP1 32 bits. The snapshot of the game was shown in Figure 4. 


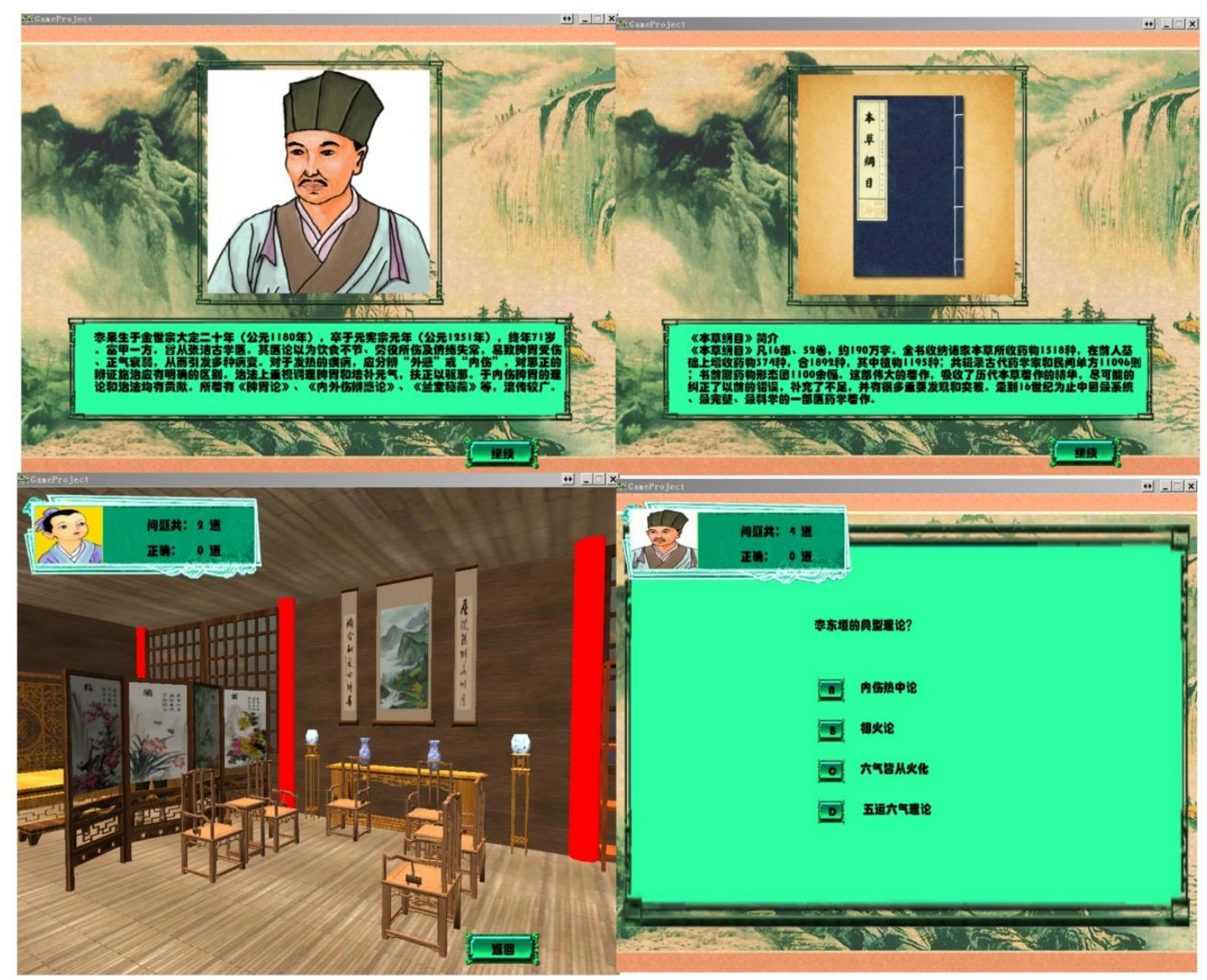

Figure 4. The Snapshot of the Game

After a series of the tests, the participants also were asked to fill out a short satisfaction questionnaire. Our findings have been quite encouraging and promising, demonstrating the viability of our methodology. A total of 100 individuals participated in this experiment and were invited to test the game. Participants can be individually classified according to different dimensions, namely whether they are regular game players and whether they had previous knowledge of the TCM. According to these characteristics, four groups were considered. The different groups were labeled A, B, C and D as follows:

- A - previous TCM knowledge and frequent game players;

- B - previous TCM knowledge and little experience with games;

- $\mathrm{C}$ - no previous TCM knowledge and frequent game players;

- D - no previous TCM knowledge and little experience with games;

Table 2. Participant Group

\begin{tabular}{cccc}
\hline Group & Frequent game player & Previous TCM knowledge & Population \\
\hline A & Yes & Yes & 13 \\
B & No & Yes & 14 \\
C & Yes & No & 17 \\
D & No & No & 56 \\
\hline
\end{tabular}


By using this game, the participants will be more familiar with the knowledge of TCM. We get some following feedback information through the investigation of 100 participants. $86 \%$ of the participants think the interactivity of the game is more advantageous to the knowledge of the communication. $77 \%$ of the participants think the game is more suitable for auxiliary teaching. $22 \%$ of the participants think the game is more suitable for autonomic learning.

\section{Discussion}

The obtained results provide a first indication that the game can provide a positive contribution to the learning of TCM. Based on a self-assessment of the students, the games help to study and remember the knowledge of TCM. The evaluation of the game also confirmed the hypothesis that the educational game highly motivates the students and provides a very positive experience. The students participated actively in the game and expressed that playing the game was an enjoyable experience. The students also demonstrated and confirmed a high degree of immersion and fun throughout the game.

\section{Main Conclusion and Future Work}

Serious gaming is being increasingly considered for use in education, training and certification for professionals. It potentially allow for the creation of scenarios that cannot be easily realized in the real world or through traditional simulation. Hence, it has the potential to actively stimulate the learning in a flexible and fun way. In this paper, we introduce how to design and implement a knowledge-intensive serious game for Education of TCM. The results of the present study indicate that the game can contribute to the learning of the TCM acknowledgement on the cognitive levels of remembering, understanding and application. Through playing the game, it can stimulate the student's interest in learning TCM acknowledgement.

Our study also has some limitations. This study was designed to be preliminary and the sample too small to offer useful data on TCM learning, but the present results offer initial evidence that the game has proven to be an engaging instructional strategy, keeping students on the task and attentive. In this respect, the game offers a possibility to complement traditional instructional strategies for teaching TCM.

In order to further generalize and to strengthen the validity of the results, it is important to obtain further evaluations. The next steps in this study include the improvement of the prototype featuring. We also intend to include other TCM discipline acknowledgement, such as acupuncture. In conclusion, we are confident that we can design and development more effective serious games in TCM education.

\section{Acknowledgements}

This publication reflects the views only of the authors, and we would like to thank the anonymous reviewers for their valuable comments. We also would like to thank the students and teachers who took part in the study. This work was supported by Beijing University of Chinese Medicine (Project No.2014-QNJSZX-022).

\section{Competing Interests}

The authors declare that they have no competing interests. 


\section{Reference}

[1] E. M. Williamson, A. Lorenc, A. Booker and N. Robinson, "The rise of traditional Chinese medicine and its materia medica: A comparison of the frequency and safety of materials and species used in Europe and China," Journal of Ethnopharmacology, vol. 149, (2013), pp. 453-462.

[2] M. Prensky, "Digital game-based learning," Computers in Entertainment (CIE), vol. 1, (2003), pp. 21-21.

[3] S. Wallace, S. Parsons, A. Westbury, K. White, K. White and A. Bailey, "Sense of presence and atypical social judgments in immersive virtual environments. Responses of adolescents with Autism Spectrum Disorders," Autism, vol. 14, (2010), pp. 199-213.

[4] A. Albin-Clark, T. L. J. Howard and B. Anderson, "Real-time computer graphics simulation of blockplay in early childhood," Computers \& Education, vol. 57, (2011), pp. 2496-2504.

[5] L. A. Annetta, J. Minogue, S. Y. Holmes and M.-T. Cheng, "Investigating the impact of video games on high school students' engagement and learning about genetics," Computers \& Education, vol. 53, (2009), pp. 74 85.

[6] B. Chang, L. Sheldon, M. Si and A. Hand, Foreign language learning in immersive virtual environments, (2012).

[7] V. Armstrong and S. Curran, "Developing a collaborative model of research using digital video," Computers \& Education, vol. 46, (2006), pp. 336-347.

[8] C. C. Abt, "Serious Games", The Viking Press, (1970).

[9] S. Chen, Z. Pan, M. Zhang and H. Shen, "A case study of user immersion-based systematic design for serious heritage games," Multimedia Tools and Applications, vol. 62, (2013), pp. 633-658.

[10] W. O. De Morais and N. Wickstrom, "A serious computer game to assist Tai Chi training for the elderly," 2011 IEEE 1st International Conference on Serious Games and Applications for Health, SeGAH, (2011) November 16-18, Braga, Portugal.

[11] D. Schwarz, P. Stourac, M. Komenda, H. Harazim, M. Kosinova, J. Gregor, R. Hulek, O. Smekalova, I. Krikava, R. Stoudek, and L. Dusek, "Interactive Algorithms for Teaching and Learning Acute Medicine in the Network of Medical Faculties MEFANET," Journal of medical Internet research, vol. 15, pp. e135-e135 (2013).

[12] Z. Michael, From Visual Simulation to Virtual Reality to Games (2005).

[13] M. Zhang, M. Xu, L. Han, Y. Liu, P. Lv and G. He, "Virtual Network Marathon with immersion, scientificalness, competitiveness, adaptability and learning," Computers \& Graphics-Uk, vol. 36, (2012), pp. 185-192.

[14] K. Starks, "Cognitive behavioral game design: a unified model for designing serious games," Frontiers in Psychology, vol. 5 (2014).

[15] A. Cetin, "3D Web Based Learning of Medical Equipments Employed in Intensive Care Units," Journal of Medical Systems, vol. 36, (2012), pp. 167-174.

[16] P. Dieckmann, "Debriefing Olympics-A Workshop Concept to Stimulate the Adaptation of Debriefings to Learning Contexts," Simulation in Healthcare-Journal of the Society for Simulation in Healthcare, vol. 7, (2012), pp. 176-182.

[17] J.-H. Chen, T.-H. Wang, W.-C. Chang, L. R. Chao and T. K. Shih, "Developing an interactive video gamebased learning environment," Journal of Software, vol. 4, (2009), pp. 132-139.

[18] I. Tarnanas, W. Schlee, M. Tsolaki, R. Müri, U. Mosimann and T. Nef, "Ecological Validity of Virtual Reality Daily Living Activities Screening for Early Dementia: Longitudinal Study," Journal of Medical Internet Research, vol. 15, (2013).

[19] A. F. S. Barbosa and F. G. M. Silva, "OxyBlood: A serious game in WebGL," 6th Iberian Conference on Information Systems and Technologies, CISTI, (2011) June 15-18, Chaves, Portugal.

[20] L. D. de Wit-Zuurendonk and S. G. Oei, "Serious gaming in women's health care," Bjog-an International Journal of Obstetrics and Gynaecology, vol. 118, (2011).

[21] S. McCallum, "Gamification and serious games for personalized health," Studies in health technology and informatics, vol. 177, (2012), pp. 85-96.

[22] S. Parsons, A. Leonard and P. Mitchell, "Virtual environments for social skills training: comments from two adolescents with autistic spectrum disorder," Computers \& Education, vol. 47, pp. 186-206 (2006).

[23] J. A. Carvalho, "Virtual Reality and ophthalmology," Revista Brasileira De Oftalmologia, vol. 71, (2012), pp. 40-47.

[24] I. Perera, C. Allison and A. Miller, "User training for 3D virtual worlds: An evaluation of training approaches," UKSim 15th International Conference on Computer Modelling and Simulation, UKSim 2013, (2013) April 10-12, pp. 129-134,Cambridge, United kingdom.

[25] J. Ribeiro, J. E. Almeida, R. J. F. Rossetti, A. Coelho and A. L. Coelho, Using Serious Games to Train Evacuation Behaviour, (2012).

[26] U. Jaffer, N. W. John and N. Standfield, "Surgical trainee opinions in the United Kingdom regarding a threedimensional virtual mentoring environment (MentorSL) in Second Life: Pilot study," JMIR Serious Games, vol. 1, (2013), pp. e2.

[27] A. F. S. Barbosa and F. G. M. Silva, "Serious Games - Design and development of OxyBlood," 8th International Conference on Advances in Computer Entertainment Technology, ACE, Ydreams; Ordem dos Engenheiros; Cent. Inf. Tecnol. Inf. (CITI)- Dep. Inf./FCT/UNL; Fund. Cienc. Tecnol. (FCT) - Minist. Cienc, 
Tecnol. Ensino Super, (2011) November 8-11, Lisbon, Portugal.

[28] T. King, M. Rosenberg, R. Braham, R. Ferguson and B. Dawson, "Life after the game - Injury profile of past elite Australian Football players," Journal of Science and Medicine in Sport, vol. 16, (2013), pp. 302-306.

[29] T. Leahey and J. Rosen, "DietBet: A Web-Based Program that Uses Social Gaming and Financial Incentives to Promote Weight Loss," JMIR Serious Games, vol. 2, (2014) pp. e2.

[30] H.-J. Chen and C.-C. Su, "Constructing a 3D virtual world for foreign language learning based on open source freeware," 6th International Conference on E-Learning and Games, Edutainment, (2011) September 7 9, pp. 46-53, Taipei, Taiwan.

[31] G.-J. Hwang, L.-H. Yang and S.-Y. Wang, "A concept map-embedded educational computer game for improving students' learning performance in natural science courses," Computers \& Education, vol. 69, (2013) pp. 121-130.

[32] J. C. Lester, H. A. Spires, J. L. Nietfeld, J. Minogue, B. W. Mott and E. V. Lobene, "Designing game-based learning environments for elementary science education: A narrative-centered learning perspective," Information Sciences, vol. 264, (2014), pp. 4-18.

[33] M. Cavazza and F. Charles, "Towards interactive narrative medicine," Studies in health technology and informatics, vol. 184, (2013), pp. 59-65.

[34] Z. Lv, A. Tek, F. Da Silva, C. Empereur-mot, M. Chavent and M. Baaden, "Game On, Science - How Video Game Technology May Help Biologists Tackle Visualization Challenges," PLoS ONE, vol. 8, (2013), pp. e57990.

[35] P. Molins-Ruano, C. Sevilla, S. Santini, P. A. Haya, P. Rodriguez and G. M. Sacha, "Designing videogames to improve students' motivation," Computers in Human Behavior, vol. 31, (2014), pp. 571-579. 
International Journal of Hybrid Information Technology Vol.8, No.3 (2015) 